Case Report

\title{
Prolonged Survival of Acute Lymphoblastic Leukemia with Intrathecal Treatments for Isolated Central Nervous System Relapse
}

\author{
Elan Gorshein $\mathbb{D}^{1},{ }^{1}$ Sheila Kalathil, ${ }^{2}$ and Mecide Gharibo ${ }^{3}$ \\ ${ }^{1}$ Rutgers Robert Wood Johnson Medical School, 3rd Floor, New Brunswick, NJ, USA \\ ${ }^{2}$ Hackensack Meridian Health Jersey Shore University, 1945 Route 33, Ackerman Building, 3rd Floor, Neptune, NJ 07753, USA \\ ${ }^{3}$ Bayer U.S. LLC, 100 Bayer Boulevard, Whippany, NJ 07891-0915, USA
}

Correspondence should be addressed to Elan Gorshein; elan.gorshein@gmail.com

Received 12 December 2017; Revised 29 December 2017; Accepted 3 January 2018; Published 31 January 2018

Academic Editor: Massimo Gentile

Copyright ( $\odot 2018$ Elan Gorshein et al. This is an open access article distributed under the Creative Commons Attribution License, which permits unrestricted use, distribution, and reproduction in any medium, provided the original work is properly cited.

Acute lymphoblastic leukemia is commonly cured when diagnosed in the pediatric population. It portends a poorer prognosis if present in adult patients. Although adults frequently achieve complete remission, relapse rates are substantial, particularly among the elderly and high-risk populations. In the absence of prophylactic intrathecal chemotherapy, more than half of patients may develop CNS involvement or relapse, which is associated with significant risk for systemic illness. This report describes a patient with acute lymphoblastic leukemia with repeated isolated CNS relapses. This case should remind clinicians that isolated CNS disease in the absence of systemic recurrence could successfully respond to intrathecal therapy and offer patients a favorable quality of life.

\section{Introduction}

Despite the overall very poor prognosis of isolated CNS relapse in acute lymphoblastic leukemia, long-term remission (in the absence of systemic relapse) may be obtained with intrathecal chemotherapy. We present a patient case that has maintained an excellent quality of life for more than eight years in the setting of greater than eighty intrathecal treatments. Limited data exist in regard to prolonged intrathecal courses in acute lymphoblastic leukemia patients, and, to our knowledge, this is the first description of successful treatment for isolated CNS relapse in the absence of systemic recurrence in this disease.

\section{Case Presentation}

The patient is a 70-year-old male with a past medical history of hypertension and dyslipidemia, who presented in September 2009 with progressive fatigue. He was noted to have a white blood cell count of 3.3 thousand/ul, with $22 \%$ circulating blasts. His staging bone marrow biopsy showed a hypercellular marrow, 95\% cellularity with complete replacement by lymphoid blasts, with flow study detecting $84 \%$ blasts expressing dim CD45 with $\operatorname{dim} \mathrm{TdT}, \mathrm{CD} 19, \mathrm{CD} 10$, CD79A, CD22, HLA-DR, and variable intensity for CD20, and CD34. The diagnosis was consistent with pre-B acute lymphoblastic leukemia, and the FISH study was negative for BCR-ABL, as well as 11Q23 translocation. His initial cytogenetic study showed a normal male karyotype. Incidentally, he was noted to have a small subacute frontoparietal subdural hematoma. Initial CSF evaluation was negative for leukemic blasts.

Our patient began induction therapy on CALGB-9111 protocol. He was noted to have persistent disease after the initial induction therapy and was transitioned to HyperCVAD part B with rituximab. He received intrathecal chemotherapy with methotrexate and was noted to have CNS leukemia involvement in November 2009. A recovery bone marrow biopsy in December 2009 showed complete remission, with adequate cellularity and normal trilineage 
hematopoiesis without residual leukemia, and normal male karyotype. He began four weekly intrathecal therapy sessions for CNS leukemia. Repeat CSF cytology studies were negative. He was deemed a poor candidate for allogeneic stem cell transplantation secondary to his age and comorbidities. In January 2010, the patient began an R-CHOP regimen, as he experienced significant complications from the Hyper-CVAD therapy (Stenotrophomonas maltophilia bacteremia with neutropenic fevers). A bone marrow biopsy after four cycles of R-CHOP showed a regenerative marrow with no evidence of residual leukemia and normal male karyotype. He then received one year of maintenance treatment with R-POMP and intrathecal chemotherapy, at which point a bone marrow biopsy in April 2011 showed no evidence of leukemia.

CSF studies at that time showed no leukemia. He then was started on maintenance therapy with rituximab therapy every two months for six cycles and intrathecal therapy every four months. He completed the course of treatment in February 2012. He had stable peripheral blood counts with no evidence of relapsed disease noted with ongoing CR1 status. In March 2013, he experienced proximal muscle weakness. MRI of his cervical, thoracic, and lumbar spine revealed no evidence of disease involvement. A diagnostic lumbar puncture for evaluation of CNS leukemia confirmed relapsed CNS disease, where the CSF fluid demonstrated a glucose level of less than $2 \mathrm{mg} /$ with over 2700 nucleated cells, the majority of which were blasts consistent with pre-B acute lymphoblastic leukemia, expressing CD19, CD20, CD10, TDT, and CD34. A subset of the cells appeared to express dim CD2 with no other apparent antigen expression noted. The flow study detected $98 \%$ of the cells were blasts. An MRI showed no leptomeningeal involvement, and a bone marrow biopsy was negative. He had an Ommaya reservoir placed in preparation for long-term intrathecal treatments.

A complete history of the patient's intrathecal treatments is listed in Table 1 . He began serial intrathecal chemotherapy alternating with methotrexate and cytarabine weekly followed by every two weeks. The patient was noted to have clearance of leukemia blasts, so the interval of intrathecal treatment was extended to every 2-3 months with alternating methotrexate and cytarabine. Repeat CSF cytology was negative throughout the sessions until April 2015, when an isolated CNS relapse was noted with no clinical signs or symptoms of systemic relapse. At that point, the patient's intrathecal chemotherapy interval was shortened to weekly therapy. CSF studies revealed no disease involvement. However, as treatment was prolonged to monthly administration, recurrent isolated CNS relapses were observed. The patient once again cleared the CSF cytology with more frequent intrathecal chemotherapy. An MRI of the brain in December 2015 showed no leptomeningeal disease. In September 2016, CSF cytology confirmed relapsed disease, but peripheral counts remained normal. His chemotherapy at that time remained with cytarabine and alternating methotrexate. He was transitioned to thiotepa in March 2017 following positive cytology. His repeat CSF studies were negative for leukemia, but in April 2017, the patient experienced another episode of isolated CNS relapse. He was
TABle 1: Chemotherapy number of IT treatments total dose (mg).

\begin{tabular}{lcc}
\hline Methotrexate $10 \mathrm{mg}$ & 2 & $20 \mathrm{mg}$ \\
Methotrexate $12 \mathrm{mg}$ & 45 & $540 \mathrm{mg}$ \\
Methotrexate $15 \mathrm{mg}$ & 6 & $90 \mathrm{mg}$ \\
Cytarabine $20 \mathrm{mg}$ & 2 & $40 \mathrm{mg}$ \\
Cytarabine $30 \mathrm{mg}$ & 4 & $120 \mathrm{mg}$ \\
Cytarabine liposomal 50 mg & 1 & $50 \mathrm{mg}$ \\
Cytarabine $70 \mathrm{mg}$ & 14 & $980 \mathrm{mg}$ \\
Cytarabine $75 \mathrm{mg}$ & 2 & $150 \mathrm{mg}$ \\
Cytarabine $100 \mathrm{mg}$ & 4 & $400 \mathrm{mg}$ \\
Thiotepa $10 \mathrm{mg}$ & 3 & $30 \mathrm{mg}$ \\
Hydrocortisone $15 \mathrm{mg}$ & 9 & $135 \mathrm{mg}$ \\
Rituximab $10 \mathrm{mg}$ & 2 & $20 \mathrm{mg}$ \\
Rituximab $25 \mathrm{mg}$ & 5 & $125 \mathrm{mg}$ \\
\hline
\end{tabular}

Intrathecal chemotherapy received.

then switched to triple therapy with intrathecal hydrocortisone, methotrexate, and cytarabine. He thereafter also received intrathecal rituximab. During these courses of treatment, CSF studies were intermittently positive for disease recurrence without signs or symptoms of systemic disease, and interval peripheral flow cytometry has demonstrated no diagnostic evidence of circulating B-cell lymphoblastic leukemia. Clinically, the patient is maintaining his quality of life eight years after his initial diagnosis, with no focal neurologic symptoms or side effects from the intrathecal treatments and no evidence of systemic relapse to date.

\section{Discussion}

Acute lymphoblastic leukemia most commonly occurs in the pediatric population. However, it is responsible for $20 \%$ of adult leukemia cases and carries a significantly poorer prognosis than in children, with overall five-year survival rates of $30-40 \%[1,2]$. This is attributed to increased drug resistance, higher risk leukemia, medical comorbidities, and poorer treatment tolerance and compliance [3]. Among adults older than 55-60 years, which defines high-risk disease, the probability of survival decreases to $20 \%$ at three years $[4,5]$. With standard intensive induction chemotherapy, approximately $85-90 \%$ of adult patients will achieve a complete remission [6]. For elderly patients, CR rates are approximately 55-60\% [7-9].

CNS involvement at the time of acute lymphoblastic leukemia diagnosis is noted in about $6 \%$ of patients [10]. The rate of detection of CNS disease is maximized by including cytological evaluation with flow cytometry [11, 12]. In the absence of CNS prophylaxis, as many as $50-75 \%$ of patients may develop CNS disease [13]. For those who receive systematic preventive therapy, CNS relapse occurs in $2-10 \%$ of patients [14]. With systemic relapse, CNS leukemia becomes more common. However, the likelihood of an isolated CNS relapse following a remission from acute lymphoblastic leukemia is approximately 5\% with standard treatment [15]. For these patients, median overall survival is approximately 6 months, and systemic recurrence risk is high and typically within a few months [15]. 
Our patient manifested disease control in the absence of systemic recurrence with the administration of intrathecal rituximab chemotherapy. Intravenous rituximab treatment achieves CNS concentrations that are significantly lower than intrathecal dosing [16]. However, there is limited data discussing the potential role of intrathecal rituximab in acute lymphoblastic leukemia with CNS relapse. In a case series of acute lymphoblastic leukemia with CNS relapse disease, five of seven patients demonstrated a complete remission following two years of CSF fluid analysis [17]. However, these patients received prior spinal or cranial irradiation and were significantly younger (none older than age 21) than our patient [17]. More recently, a case series of pediatric patients with B-cell lymphoid CD20+ malignancies reported an ongoing complete remission in only one of three patients with acute lymphoblastic leukemia who were treated with intraventricular or intrathecal rituximab [18]. Thus, preliminary data on the use of intrathecal or intraventricular rituximab therapy for CNS involvement of CD20+ acute lymphoblastic leukemia suggest a potential benefit, but requires further evaluation in this setting.

\section{Conflicts of Interest}

The authors declare that there are no conflicts of interest regarding the publication of this article.

\section{References}

[1] R. L. Siegel, K. D. Miller, and A. Jemal, "Cancer statistics, 2017," A Cancer Journal for Clinicians, vol. 67, no. 1, pp. 7-30, 2017.

[2] A. Kansagra, S. Dahiya, and M. Litzow, "Continuing challenges and current issues in acute lymphoblastic leukemia," Leukemia \& Lymphoma, pp. 1-16, 2017.

[3] C. H. Pui and W. E. Evans, "Treatment of acute lymphoblastic leukemia," New England Journal of Medicine, vol. 354, no. 2, pp. 166-178, 2006.

[4] R. Bassan and D. Hoelzer, "Modern therapy of acute lymphoblastic leukemia," Journal of Clinical Oncology, vol. 29, no. 5, pp. 532-543, 2011.

[5] S. Faderl, S. O’Brien, C. H. Pui et al., "Adult acute lymphoblastic leukemia: concepts and strategies," Cancer, vol. 116, no. 5, pp. 1165-1176, 2010.

[6] N. Gökbuget, M. Kneba, T. Raff et al., "Adult patients with acute lymphoblastic leukemia and molecular failure display a poor prognosis and are candidates for stem cell transplantation and targeted therapies," Blood, vol. 120, no. 9, pp. 1868-1876, 2012.

[7] N. Gokbuget, J. Beck, and M. Bruggemann, "Moderate intensive chemotherapy including CNS prophylaxis with liposomal cytarabine is feasible and effective in older patients with $\mathrm{Ph}$ - negative acute lymphoblastic leukemia (ALL): results of a prospective trial from the German Multicenter Study Group for Adult ALL (GMALL)," Blood, vol. 120, p. 1493, 2012.

[8] N. Gökbuget, "How I treat older patients with ALL," Blood, vol. 122, no. 8, pp. 1366-1375, 2013.

[9] A. Delannoy, B. Cazin, X. Thomas et al., "Treatment of acute lymphoblastic leukemia in the elderly: an evaluation of interferon alpha given as a single agent after complete remission," Leukemia \& Lymphoma, vol. 43, no. 1, pp. 75-81, 2002.
[10] J. Dass, A. Dayama, P. C. Mishra et al., "Higher rate of central nervous system involvement by flow cytometry than morphology in acute lymphoblastic leukemia," International Journal of Laboratory Hematology, vol. 39, no. 5, pp. 546-551, 2017.

[11] H. M. Lazarus, S. M. Richards, R. Chopra et al., "Central nervous system involvement in adult acute lymphoblastic leukemia at diagnosis: results from the international ALL trial MRC UKALL-XII/ECOG E2993," Blood, vol. 108, no. 2, pp. 465-472, 2006.

[12] J. E. Bromberg, D. A. Breems, J. Kraan et al., "CSF flow cytometry greatly improves diagnostic accuracy in CNS hematologic malignancies," Neurology, vol. 68, no. 20, pp. 1674-1679, 2007.

[13] U. R. Surapaneni, J. E. Cortes, D. Thomas et al., "Central nervous system relapse in adults with acute lymphoblastic leukemia," Cancer, vol. 94, no. 3, pp. 773-779, 2002.

[14] C. H. Pui, "Central nervous system disease in acute lymphoblastic leukemia: prophylaxis and treatment," Hematology, vol. 2006, no. 1, pp. 142-146, 2006.

[15] J. M. Sancho, J. M. Ribera, A. Oriol et al., "Central nervous system recurrence in adult patients with acute lymphoblastic leukemia: frequency and prognosis in 467 patients without cranial irradiation for prophylaxis," Cancer, vol. 106, no. 12, pp. 2540-2548, 2006.

[16] A. J. Perissinotti and D. J. Reeves, "Role of intrathecal rituximab and trastuzumab in the management of leptomeningeal carcinomatosis," Annals of Pharmacotherapy, vol. 44, no. 10, pp. 1633-1640, 2010.

[17] J. C. Jaime-Pérez, L. N. Rodríguez-Romo, O. González-Llano, A. Chapa-Rodríguez, and D. Gómez-Almaguer, "Effectiveness of intrathecal rituximab in patients with acute lymphoblastic leukaemia relapsed to the CNS and resistant to conventional therapy," British Journal of Haematology, vol. 144, no. 5, pp. 794-795, 2009.

[18] F. Ceppi, S. Weitzman, W. Woessmann et al., "Safety and efficacy of intrathecal rituximab in children with B cell lymphoid CD20+ malignancies: An international retrospective study," American Journal of Hematology, vol. 91, no. 5, pp. 486-491, 2016. 


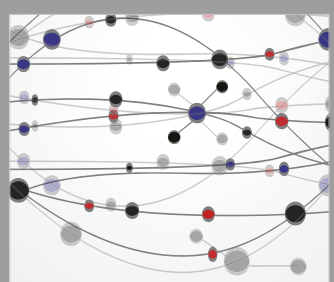

The Scientific World Journal
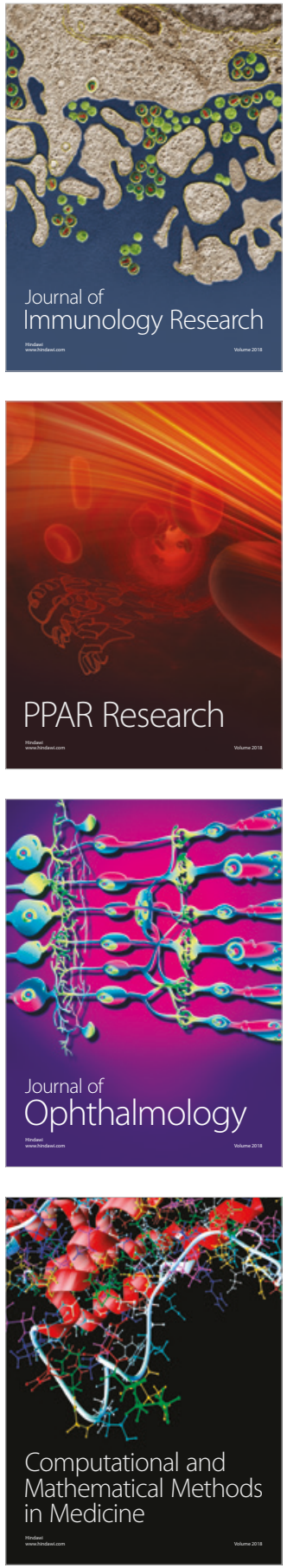

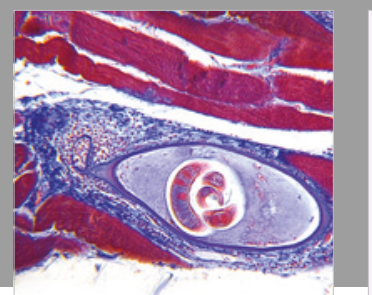

Gastroenterology Research and Practice

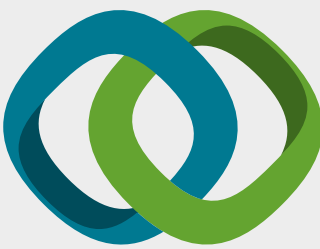

\section{Hindawi}

Submit your manuscripts at

www.hindawi.com
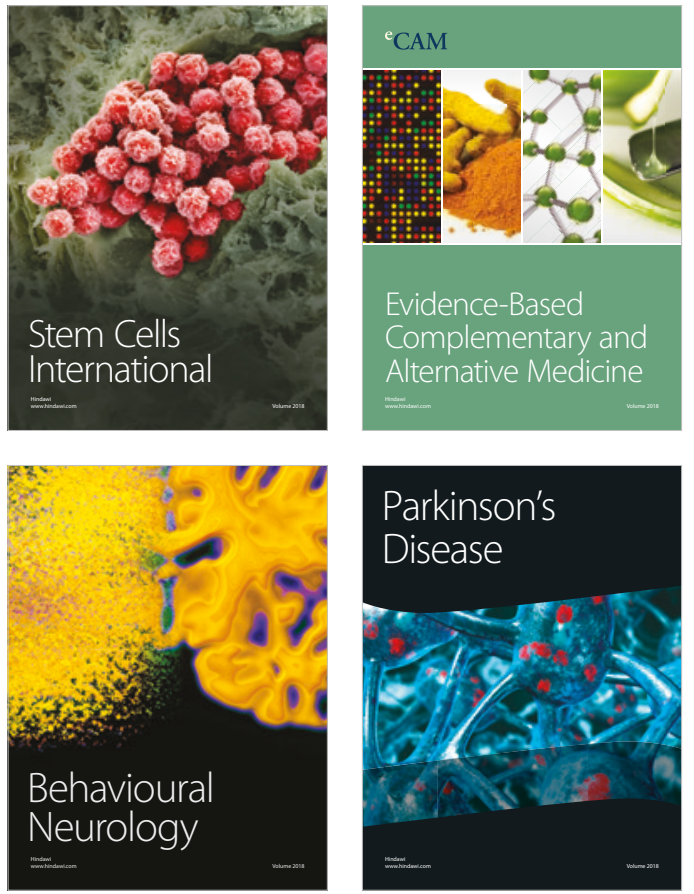

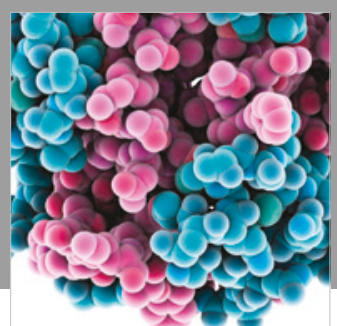

ournal of

Diabetes Research

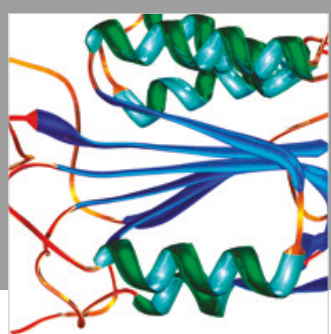

Disease Markers
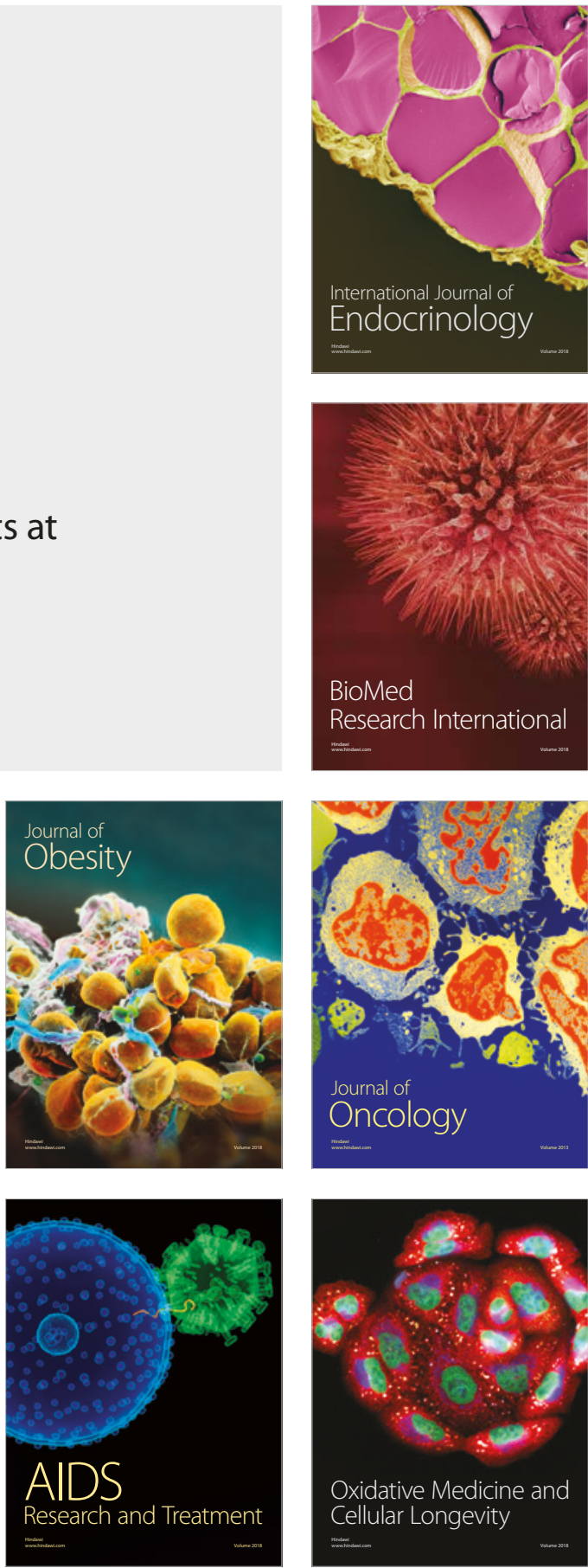